\title{
On the Enumeration and Congruences for $m$-ary Partitions
}

\author{
Lisa Hui Sun ${ }^{1}$ and Mingzhi Zhang ${ }^{2}$ \\ Center for Combinatorics, LPMC \\ Nankai University, Tianjin 300071, P. R. China \\ ${ }^{1}$ sunhui@nankai.edu.cn, ${ }^{2}$ mingzhi@mail.nankai.edu.cn
}

\begin{abstract}
Let $m \geq 2$ be a fixed integer. Suppose that $n$ is a positive integer such that $m^{j} \leq n<m^{j+1}$ for some integer $j \geq 0$. Denote $b_{m}(n)$ the number of $m$-ary partitions of $n$, where each part of the partition is a power of $m$. In this paper, we show that $b_{m}(n)$ can be represented as a $j$-fold summation by constructing a one-to-one correspondence between the $m$-ary partitions and a special class of integer sequences relying only on the base $m$ representation of $n$. It directly reduces to Andrews, Fraenkel and Sellers' characterization of the values $b_{m}(m n)$ modulo $m$. Moreover, denote $c_{m}(n)$ the number of $m$-ary partitions of $n$ without gaps, wherein if $m^{i}$ is the largest part, then $m^{k}$ for each $0 \leq k<i$ also appears as a part. We also obtain an enumeration formula for $c_{m}(n)$ which leads to an alternative representation for the congruences of $c_{m}(m n)$ modulo $m$ due to Andrews, Fraenkel and Sellers.
\end{abstract}

Keywords: $m$-ary partition, base $m$ representation, congruence

AMS Classification: 05A17, 11P83

\section{Introduction}

The arithmetic properties for partition functions have been extensively studied since the discoveries of Ramanujan [12. In this paper, we are mainly concerned with the enumeration of $m$-ary partitions which leads to the congruence properties given by Andrews, Fraenkel and Sellers [3, 4].

Let $m \geq 2$ be a fixed integer. An $m$-ary partition of a positive integer $n$ is a partition of $n$ such that each part is a power of $m$. The number of $m$-ary partitions of $n$ is denoted by $b_{m}(n)$. For example, there are five 3 -ary partitions for $n=10$ :

$$
9+1,3+3+3+1,3+3+1+1+1+1,3+1+1+1+1+1+1+1,1+1+1+1+1+1+1+1+1+1 .
$$

Thus, $b_{3}(10)=5$. Denote an $m$-ary partition of $n$ by a sequence $\lambda=\left(a_{\ell}, a_{\ell-1}, \ldots, a_{0}\right)$ such that

$$
n=a_{\ell} m^{\ell}+a_{\ell-1} m^{\ell-1}+\cdots+a_{0}
$$


where $a_{\ell}>0$ and $a_{i} \geq 0$ for $0 \leq i \leq \ell-1$. Denote the set of all the $m$-ary partitions of $n$ by $\mathcal{B}_{m}(n)$. It is known that the generating function of $b_{m}(n)$ is given by

$$
B_{m}(q)=\sum_{n=0}^{\infty} b_{m}(n) q^{n}=\prod_{k=0}^{\infty} \frac{1}{1-q^{m^{k}}} .
$$

For the case $m=2$, Churchhouse [6] conjectured the following congruences for the binary partition function $b_{2}(n)$ :

$$
\begin{aligned}
& b_{2}\left(2^{2 k+2} n\right) \equiv b_{2}\left(2^{2 k} n\right) \quad\left(\bmod 2^{3 k+2}\right), \\
& b_{2}\left(2^{2 k+1} n\right) \equiv b_{2}\left(2^{2 k-1} n\right) \quad\left(\bmod 2^{3 k}\right),
\end{aligned}
$$

where $n, k \geq 1$. The conjecture was first proved by Rødseth [13] and further studied by Hirschhorn and Loxton [9]. Later, it was extended to $m$-ary partitions by Andrews [1, Gupta [8], and Rødseth and Sellers [14].

Throughout this paper, without specification, we set $n$ to be a positive integer such that $m^{j} \leq n<m^{j+1}$ for some integer $j \geq 0$. Recall that the base $m$ representation of $n$ is the unique expression of $n$ which can be written as follows

$$
n=\alpha_{j} m^{j}+\alpha_{j-1} m^{j-1}+\cdots+\alpha_{1} m+\alpha_{0}
$$

where $\alpha_{j}>0$ and $0 \leq \alpha_{i} \leq m-1$ for $0 \leq i \leq j-1$. Denote the base $m$ representation of $n$ by

$$
r_{m}(n)=\left(\alpha_{j}, \alpha_{j-1}, \ldots, \alpha_{1}, \alpha_{0}\right) .
$$

Based on the base $m$ representation of $n$, Andrews, Fraenkel and Sellers [3, Theorem 1] provided the following modulo $m$ characterization of $b_{m}(m n)$ :

$$
b_{m}(m n) \equiv \prod_{i=0}^{j}\left(\alpha_{i}+1\right) \quad(\bmod m)
$$

In this paper, by establishing a bijection between the set $\mathcal{B}_{m}(n)$ of $m$-ary partitions of $n$ and the set of integer sequences given in the following theorem, we derive a $j$-fold summation formula for $b_{m}(n)$. It will directly lead to Andrews, Fraenkel and Sellers' congruence (1.5).

Theorem 1.1. There is a one-to-one correspondence between the set $\mathcal{B}_{m}(n)$ of m-ary partitions of $n$ and the following set of integer sequences

$$
\mathcal{S}_{m}(n)=\left\{\left(\beta_{j}, \beta_{j-1}, \ldots, \beta_{1}\right) \mid 0 \leq \beta_{j} \leq \alpha_{j} \text { and } 0 \leq \beta_{t} \leq \alpha_{t}+m \beta_{t+1} \text { for } 1 \leq t \leq j-1\right\} .
$$

Based on the above bijection, we provide a combinatorial approach to derive the following $j$-fold summation formula for $b_{m}(n)$. 
Theorem 1.2. Let $r_{m}(n)=\left(\alpha_{j}, \alpha_{j-1}, \ldots, \alpha_{1}, \alpha_{0}\right)$ be the base $m$ representation of $n$. We have

$$
b_{m}(n)=\sum_{k_{j}=0}^{\alpha_{j}} \sum_{k_{j-1}=0}^{\alpha_{j-1}+m k_{j}} \cdots \sum_{k_{1}=0}^{\alpha_{1}+m k_{2}} 1 .
$$

Obviously, $b_{m}(n)=1$ when $j=0$.

Notice that if $r_{m}(n)=\left(\alpha_{j}, \alpha_{j-1}, \cdots, \alpha_{1}, \alpha_{0}\right)$, then $r_{m}(m n)=\left(\alpha_{j}, \alpha_{j-1}, \cdots, \alpha_{1}, \alpha_{0}, 0\right)$. Thus the above theorem leads to that

$$
b_{m}(m n)=\sum_{k_{j}=0}^{\alpha_{j}} \sum_{k_{j-1}=0}^{\alpha_{j-1}+m k_{j}} \cdots \sum_{k_{1}=0}^{\alpha_{1}+m k_{2}} \sum_{k_{0}=0}^{\alpha_{0}+m k_{1}} 1 .
$$

By taking modulo $m$ on both sides of the above equation, it directly reduces to Andrews, Fraenkel and Sellers' congruence (1.5).

We also consider the cases for the $m$-ary partitions without gaps, wherein if $m^{i}$ is the largest part, then $m^{k}$ for each $0 \leq k<i$ also appears as a part. The related works on such restricted $m$-ary partitions can be found in [2, 4, 11]. Moreover, in [5, 7, 10, 15], a general class of non-squashing partitions was introduced and studied, which contains $m$-ary partitions as a special case.

Let $c_{m}(n)$ denote the number of $m$-ary partitions without gaps of $n$. Based on the bijection given in Theorem 1.1, we also obtain the following enumeration formula for $c_{m}(n)$.

Theorem 1.3. Let $r_{m}(n)=\left(\alpha_{j}, \alpha_{j-1}, \ldots, \alpha_{1}, \alpha_{0}\right)$ be the base $m$ representation of $n$. We have

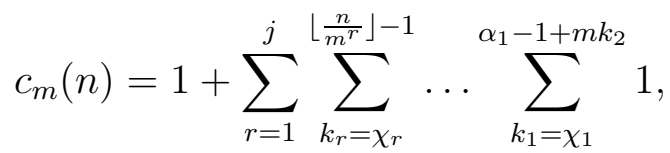

where for $1 \leq i \leq r$,

$$
\chi_{i}= \begin{cases}0, & \text { if } \alpha_{i-1}>0, \\ 1, & \text { if } \alpha_{i-1}=0 .\end{cases}
$$

Applying formula (1.7), we obtain the following congruence property of $c_{m}(m n)$, which reveals the results given by Andrews, Fraenkel and Sellers [4, Theorem 2.1].

Theorem 1.4. Let $\chi_{i}$ be defined by (1.8) for $1 \leq i \leq r$, then we have

$$
c_{m}(m n) \equiv \alpha_{0}+\left(\alpha_{0}-1\right) \sum_{i=1}^{j}\left(\alpha_{1}-\chi_{1}\right)\left(\alpha_{2}-\chi_{2}\right) \cdots\left(\alpha_{i}-\chi_{i}\right) \quad(\bmod m) .
$$




\section{The enumeration formula for $b_{m}(n)$}

In this section, we provide a bijection between the set $\mathcal{B}_{m}(n)$ of $m$-ary partitions of $n$ and the set

$$
\mathcal{S}_{m}(n)=\left\{\left(\beta_{j}, \beta_{j-1}, \ldots, \beta_{1}\right) \mid 0 \leq \beta_{j} \leq \alpha_{j} \text { and } 0 \leq \beta_{t} \leq \alpha_{t}+m \beta_{t+1} \text { for } 1 \leq t \leq j-1\right\},
$$

which relies only on the base $m$ representation of $n$. It will lead to the enumeration formula (1.6) for the $m$-ary partitions.

To this end, we first define the following subtraction between the base $m$ representation of $n$ and an ordinary $m$-ary partition of $n$.

Definition 2.1. Let $r_{m}(n)=\left(\alpha_{j}, \alpha_{j-1}, \ldots, \alpha_{0}\right)$ be the base $m$ representation of $n$ and $\lambda=\left(\lambda_{\ell}, \lambda_{\ell-1}, \ldots, \lambda_{0}\right)$ be an m-ary partition of $n$. Then subtracting $\lambda$ from $r_{m}(n)$ is given as follows

$$
r_{m}(n)-\lambda=\left(\beta_{j}, \beta_{j-1}, \ldots, \beta_{1}\right)
$$

where for $1 \leq i \leq j$,

$$
\beta_{i}=\sum_{k=i}^{j} m^{k-i}\left(\alpha_{k}-\lambda_{k}\right)
$$

provided that $\lambda_{k}=0$ for $\ell<k \leq j$.

We further show that the subtraction (2.1) defined above gives a bijection between $\mathcal{B}_{m}(n)$ and $\mathcal{S}_{m}(n)$.

Theorem 2.2. Let $r_{m}(n)=\left(\alpha_{j}, \alpha_{j-1}, \ldots, \alpha_{0}\right)$ be the base $m$ representation of $n$ and $\lambda=\left(\lambda_{\ell}, \lambda_{\ell-1}, \ldots, \lambda_{0}\right)$ be an arbitrary $m$-ary partition of $n$. Define a map $\varphi$ from $\mathcal{B}_{m}(n)$ to $\mathcal{S}_{m}(n)$ by $\varphi(\lambda)=r_{m}(n)-\lambda$. Then $\varphi$ is a bijection between $\mathcal{B}_{m}(n)$ and $\mathcal{S}_{m}(n)$.

Proof. Denote $\beta=\varphi(\lambda)=\left(\beta_{j}, \beta_{j-1}, \ldots, \beta_{1}\right)$. First, we proceed to show that $\beta \in \mathcal{S}_{m}(n)$ and thereby $\varphi$ is well defined. Following Definition 2.1 , it is easy to see that

$$
\begin{aligned}
& \beta_{j}=\alpha_{j}-\lambda_{j}, \\
& \beta_{t}=\alpha_{t}-\lambda_{t}+m \beta_{t+1},
\end{aligned}
$$

where $1 \leq t \leq j-1$ and $\lambda_{k}=0$ for $\ell<k \leq j$. Since $\lambda_{k} \geq 0$ for $0 \leq k \leq j$, we see that $\beta_{j} \leq \alpha_{j}$ and $\beta_{t} \leq \alpha_{t}+m \beta_{t+1}$ for $1 \leq t \leq j-1$.

It is obvious that $\lambda_{j} \leq \alpha_{j}$, so that $\beta_{j} \geq 0$. From the fact that

$$
\lambda_{j} m^{j}+\lambda_{j-1} m^{j-1}+\cdots+\lambda_{0}=\alpha_{j} m^{j}+\alpha_{j-1} m^{j-1}+\cdots+\alpha_{0}
$$

we are led to that for $1 \leq t \leq j-1$,

$$
\lambda_{j} m^{j}+\lambda_{j-1} m^{j-1}+\cdots+\lambda_{t} m^{t} \leq \alpha_{j} m^{j}+\alpha_{j-1} m^{j-1}+\cdots+\alpha_{0}
$$


Hence we obtain that

$$
\left(\left(\lambda_{t}-\alpha_{t}\right)+\sum_{k=1}^{j-t}\left(\lambda_{t+k}-\alpha_{t+k}\right) m^{k}\right) m^{t} \leq \alpha_{t-1} m^{t-1}+\alpha_{t-2} m^{t-2}+\cdots+\alpha_{0} .
$$

Since $\left(\alpha_{j}, \alpha_{j-1}, \ldots, \alpha_{0}\right)$ is the base $m$ representation of $n$, it is obvious that

$$
\alpha_{t-1} m^{t-1}+\alpha_{t-2} m^{t-2}+\cdots+\alpha_{0}<m^{t}
$$

which implies that

$$
\left(\left(\lambda_{t}-\alpha_{t}\right)+\sum_{k=1}^{j-t}\left(\lambda_{t+k}-\alpha_{t+k}\right) m^{k}\right) m^{t}<m^{t}
$$

Note that $\lambda_{k}$ and $\alpha_{k}$ are all integers for $0 \leq k \leq j$, it follows that

$$
\lambda_{t}-\alpha_{t}+\sum_{k=1}^{j-t}\left(\lambda_{t+k}-\alpha_{t+k}\right) m^{k} \leq 0
$$

and thereby

$$
\lambda_{t} \leq \alpha_{t}+\sum_{k=1}^{j-t}\left(\alpha_{t+k}-\lambda_{t+k}\right) m^{k}=\alpha_{t}+m \beta_{t+1}
$$

By (2.3), it directly leads to that $\beta_{t} \geq 0$ for $1 \leq t \leq j-1$. Thus $\beta \in \mathcal{S}_{m}(n)$ and $\varphi$ is well defined.

To prove that $\varphi$ is a bijection, it is sufficient to show that there exists the inverse map of $\varphi$. For a given $\beta=\left(\beta_{j}, \beta_{j-1}, \ldots, \beta_{1}\right) \in \mathcal{S}_{m}(n)$, let $\varphi^{-1}(\beta)$ be given by computing

$$
\lambda^{\prime}=\left(\alpha_{j}-\beta_{j}, \alpha_{j-1}-\beta_{j-1}+m \beta_{j}, \ldots, \alpha_{1}-\beta_{1}+m \beta_{2}, \alpha_{0}+m \beta_{1}\right)
$$

and then deleting the preceding zeros. From the definition of $\mathcal{S}_{m}(n)$, we see that each element of $\lambda^{\prime}$ is nonnegative. Furthermore, it is easy to see that

$$
\left(\alpha_{j}-\beta_{j}\right) m^{j}+\left(\alpha_{j-1}-\beta_{j-1}+m \beta_{j}\right) m^{j-1}+\cdots+\alpha_{0}+m \beta_{1}=n,
$$

which implies that $\varphi^{-1}(\beta)$ is an $m$-ary partition of $n$ and thereby $\varphi^{-1}(\beta) \in \mathcal{B}_{m}(n)$. It completes the proof of the bijection.

For example, let $m=4$ and $n=36$, then the base 4 representation of 36 is $r_{4}(36)=$ $(2,1,0)$. The correspondence between all the 4 -ary partitions of 36 and the integer sequences belonging to $\mathcal{S}_{4}(36)$ can be seen in Table 2.1 .

The above theorem directly leads to that $b_{m}(n)=\left|\mathcal{B}_{m}(n)\right|=\left|\mathcal{S}_{m}(n)\right|$. By studying the recursive properties of the sequences in $\mathcal{S}_{m}(n)$, we obtain the $j$-fold summation formula (1.6) of $b_{m}(n)$. Now we give the detailed proof of Theorem 1.2 . 
Table 2.1: The correspondence between $\lambda \in \mathcal{B}_{4}(36)$ and $\beta \in \mathcal{S}_{4}(36)$

\begin{tabular}{cccccccccc}
\hline$\lambda$ & $(2,1,0)$ & $(2,0,4)$ & $(1,5,0)$ & $(1,4,4)$ & $(1,3,8)$ & $(1,2,12)$ & $(1,1,16)$ & $(1,0,20)$ & $(0,9,0)$ \\
$\beta$ & $(0,0)$ & $(0,1)$ & $(1,0)$ & $(1,1)$ & $(1,2)$ & $(1,3)$ & $(1,4)$ & $(1,5)$ & $(2,0)$ \\
\hline$\lambda$ & $(0,8,4)$ & $(0,7,8)$ & $(0,6,12)$ & $(0,5,16)$ & $(0,4,20)$ & $(0,3,24)$ & $(0,2,28)$ & $(0,1,32)$ & $(0,0,36)$ \\
$\beta$ & $(2,1)$ & $(2,2)$ & $(2,3)$ & $(2,4)$ & $(2,5)$ & $(2,6)$ & $(2,7)$ & $(2,8)$ & $(2,9)$ \\
\hline
\end{tabular}

Proof of Theorem 1.2. Denote the summation on the right hand side of (1.6) by

$$
f\left(\alpha_{j}, \alpha_{j-1}, \ldots, \alpha_{1}, \alpha_{0}\right)=\sum_{k_{j}=0}^{\alpha_{j}} \sum_{k_{j-1}=0}^{\alpha_{j-1}+m k_{j}} \ldots \sum_{k_{2}=0}^{\alpha_{2}+m k_{3}} \sum_{k_{1}=0}^{\alpha_{1}+m k_{2}} 1 .
$$

We prove the theorem by induction. When $j=0, r_{m}(n)=\left(\alpha_{0}\right)$ with $0 \leq \alpha_{0} \leq m-1$. It is obvious that $b_{m}(n)=f\left(\alpha_{0}\right)=1$.

Suppose that (1.6) holds for $j=i-1$. When $j=i$, we have $r_{m}(n)=\left(\alpha_{i}, \alpha_{i-1}, \ldots, \alpha_{1}, \alpha_{0}\right)$. By Theorem 2.2, it implies that $b_{m}(n)=\left|\mathcal{S}_{m}(n)\right|$ where

$$
\mathcal{S}_{m}(n)=\left\{\left(\beta_{i}, \beta_{i-1}, \ldots, \beta_{1}\right) \mid 0 \leq \beta_{i} \leq \alpha_{i}, 0 \leq \beta_{t} \leq \alpha_{t}+m \beta_{t+1}, 1 \leq t \leq i-1\right\} .
$$

For a fixed $\beta_{i}$ with $0 \leq \beta_{i} \leq \alpha_{i}$, let us consider the subset of $\mathcal{S}_{m}(n)$ with $\beta_{i}$ being the first entry. By deleting $\beta_{i}$ in these sequences, it is easy to see that this subset is bijective with the following set

$$
\mathcal{S}_{\beta_{i}}=\left\{\left(\beta_{i-1}, \beta_{i-2}, \ldots, \beta_{1}\right) \mid 0 \leq \beta_{t} \leq \alpha_{t}+m \beta_{t+1}, 1 \leq t \leq i-1\right\},
$$

and therefore

$$
\mathcal{S}_{m}(n)=\bigcup_{\beta_{i}=0}^{\alpha_{i}} \mathcal{S}_{\beta_{i}}
$$

By induction, we obtain that the cardinality of the set $\mathcal{S}_{\beta_{i}}$ is

$$
\left|\mathcal{S}_{\beta_{i}}\right|=f\left(\alpha_{i-1}+m \beta_{i}, \alpha_{i-1}, \ldots, \alpha_{0}\right)=\sum_{k_{i-1}=0}^{\alpha_{i-1}+m \beta_{i}} \ldots \sum_{k_{2}=0}^{\alpha_{2}+m k_{3}} \sum_{k_{1}=0}^{\alpha_{1}+m k_{2}} 1 .
$$

Then by summing the above equation for $\beta_{i}$ from 0 to $\alpha_{i}$, we obtain

$$
\left|\mathcal{S}_{m}(n)\right|=f\left(\alpha_{i}, \alpha_{i-1}, \ldots, \alpha_{0}\right)=\sum_{k_{i}=0}^{\alpha_{i}} \sum_{k_{i-1}=0}^{\alpha_{i-1}+m k_{i}} \cdots \sum_{k_{2}=0}^{\alpha_{2}+m k_{3}} \sum_{k_{1}=0}^{\alpha_{1}+m k_{2}} 1
$$

which completes the proof.

Note that the $j$-fold summation formula (1.6) also can be derived from the generating function (1.1) of $b_{m}(n)$. Moreover, by setting $M=\{m, m, \ldots\}$ in the summation given by Folsom, Homma, Ryu and Tong [7, Theorem 1.5], it reduces to another $j$-fold summation expression for $b_{m}(n)$. 


\section{The $m$-ary partitions without gaps}

In this section, based on the bijection given in Theorem 2.2, we derive an enumeration formula for the $m$-ary partitions without gaps. Denote $c_{m}(n)$ the number of this restricted $m$-ary partitions of $n$. We also obtain an alternative expression for the congruence properties of $c_{m}(m n)$ given by Andrews, Fraenkel and Sellers [4, Theorem 2.1].

Recall that by using the base $m$ representation of $n$ in the following form

$$
n=\sum_{i=\ell}^{\infty} \alpha_{i} m^{i}
$$

where $1 \leq \alpha_{\ell}<m$ and $0 \leq \alpha_{i}<m$ for $i>\ell$, Andrews, Fraenkel and Sellers obtained the following result.

Theorem 3.1 (Andrews, Fraenkel and Sellers [4, Theorem 2.1]). (1) If $\ell$ is even, then

$$
c_{m}(m n) \equiv \alpha_{\ell}+\left(\alpha_{\ell}-1\right) \sum_{i=\ell+1}^{\infty} \alpha_{\ell+1} \cdots \alpha_{i} \quad(\bmod m) .
$$

(2) If $\ell$ is odd, then

$$
c_{m}(m n) \equiv 1-\alpha_{\ell}-\left(\alpha_{\ell}-1\right) \sum_{i=\ell+1}^{\infty} \alpha_{\ell+1} \cdots \alpha_{i} \quad(\bmod m) .
$$

Denote the floor function of a real number $a$ by $\lfloor a\rfloor$, which is the largest integer less than or equal to $a$. To derive our expression of the congruences (3.1) and (3.2), first let us show how to derive the enumeration formula (1.7) for $c_{m}(n)$ as given in Theorem 1.3.

Proof of Theorem 1.3. Denote the set of all the $m$-ary partitions without gaps of $n$ by $\mathcal{G}_{m}(n)$. We claim that for any $\lambda \in \mathcal{G}_{m}(n)$, it can be written as

$$
\lambda=\left(\left\lfloor\frac{n}{m^{r}}\right\rfloor-\beta_{r}, \alpha_{r-1}-\beta_{r-1}+m \beta_{r}, \ldots, \alpha_{0}+m \beta_{1}\right),
$$

where $0 \leq r \leq j$ and $\beta_{i}$ are integers such that

$$
\left\lfloor\frac{n}{m^{r}}\right\rfloor-\beta_{r}>0, \alpha_{r-1}-\beta_{r-1}+m \beta_{r}>0, \ldots, \alpha_{0}+m \beta_{1}>0 .
$$

Specially, when $r=0$, there is a unique $m$-ary partition without gaps, say, $\lambda=(n)$ which consists of $n$ ones. For $r>1$, as an example, we consider $m=4$ and $n=73$, then $r_{4}(73)=(1,0,2,1)$. When $r=2$, we can obtain that $(3,6,1)$ is a 4 -ary partition without gaps which can be represented as $\left(\left\lfloor\frac{73}{4^{2}}\right\rfloor-1,2-0+4 \times 1,1+4 \times 0\right)$.

Recall that

$$
n=\alpha_{j} m^{j}+\alpha_{j-1} m^{j-1}+\cdots+\alpha_{0}
$$


For $1 \leq r \leq j$, it follows that

$$
\begin{aligned}
& \left(\left\lfloor\frac{n}{m^{r}}\right\rfloor-\beta_{r}\right) m^{r}+\left(\alpha_{r-1}-\beta_{r-1}+m \beta_{r}\right) m^{r-1}+\cdots+\left(\alpha_{1}-\beta_{1}+m \beta_{2}\right) m+\alpha_{0}+m \beta_{1} \\
& \quad=\left\lfloor\frac{n}{m^{r}}\right\rfloor m^{r}+\alpha_{r-1} m^{r-1}+\cdots+\alpha_{1} m+\alpha_{0} \\
& \quad=\left(\alpha_{j} m^{j-r}+\cdots+\alpha_{r}\right) m^{r}+\alpha_{r-1} m^{r-1}+\cdots+\alpha_{1} m+\alpha_{0} \\
& \quad=\alpha_{j} m^{j}+\cdots+\alpha_{r} m^{r}+\alpha_{r-1} m^{r-1}+\cdots+\alpha_{1} m+\alpha_{0} \\
& \quad=n
\end{aligned}
$$

which certifies that $\lambda \in \mathcal{G}_{m}(n)$. For a fixed integer $r$ such that $0 \leq r \leq j$, by the bijection given in Theorem 2.2, we get

$$
\varphi(\lambda)=\left(\alpha_{j}, \alpha_{j-1}+m \alpha_{j}, \ldots, \sum_{k=r+1}^{j} m^{k-r-1} \alpha_{k}, \beta_{r}, \beta_{r-1}, \ldots, \beta_{1}\right) .
$$

Note that for any $\lambda \in \mathcal{G}_{m}(n)$ with the given $r$, the first $j-r$ elements in $\varphi(\lambda)$ are the same, which only depend on $\alpha_{r+1}, \ldots, \alpha_{j}$. Then by deleting these terms, we find that the set of $m$-ary partitions without gaps $\mathcal{G}_{m}(n)$ is in one-to-one correspondence with the following set of integer sequences

$$
\mathcal{R}_{m}(n)=\bigcup_{r=0}^{j}\left\{\left(\beta_{r}, \ldots, \beta_{1}\right) \mid\left\lfloor\frac{n}{m^{r}}\right\rfloor-\beta_{r}>0, \alpha_{r-1}-\beta_{r-1}+m \beta_{r}>0, \ldots, \alpha_{0}+m \beta_{1}>0\right\} .
$$

It indicates that $c_{m}(n)=\left|\mathcal{R}_{m}(n)\right|$.

From the conditions (3.3), we see that if $\alpha_{0}=0$, then $\beta_{1}>0$, which means $\beta_{1}$ starts from 1 . If $\alpha_{0}>0$, then $\beta_{1} \geq 0$, which means $\beta_{1}$ starts from 0 . For both cases, we denote $\beta_{1}$ starting from $\chi_{1}$, which is defined by (1.8). By $\alpha_{1}-\beta_{1}+m \beta_{2}>0$ we have $\beta_{1}<\alpha_{1}+m \beta_{2}$. Thereby we see that $\beta_{1}$ ranges from $\chi_{1}$ to $\alpha_{1}+m \beta_{2}-1$. By similar arguments applying to $\beta_{i}$ for $2 \leq i \leq r$, we have

$$
c_{m}(n)=\left|\mathcal{R}_{m}(n)\right|=1+\sum_{r=1}^{j} \sum_{k_{r}=\chi_{r}}^{\left\lfloor\frac{n}{\left.m^{r}\right\rfloor-1} \cdots\right.} \sum_{k_{1}=\chi_{1}}^{\alpha_{1}-1+m k_{2}} 1
$$

where for $1 \leq i \leq j$,

$$
\chi_{i}= \begin{cases}0, & \text { if } \alpha_{i-1}>0 \\ 1, & \text { if } \alpha_{i-1}=0\end{cases}
$$

This completes the proof.

Noting that $r_{m}(m n)=\left(\alpha_{j}, \alpha_{j-1}, \ldots, \alpha_{0}, 0\right)$, then by applying the above result we have

$$
c_{m}(m n)=1+\sum_{r=1}^{j+1} \sum_{k_{r}=\chi_{r-1}}^{\left\lfloor\frac{m n}{m^{r}}\right\rfloor-1} \ldots \sum_{k_{2}=\chi_{1}}^{\alpha_{1}-1+m k_{3}} \sum_{k_{1}=1}^{\alpha_{0}-1+m k_{2}} 1 .
$$


By taking modulo $m$ on both sides of the above identity, we directly obtain the congruence property (1.9), namely,

$$
\begin{aligned}
c_{m}(m n) & \equiv 1+\sum_{r=1}^{j+1}\left(\alpha_{0}-1\right)\left(\alpha_{1}-\chi_{1}\right)\left(\alpha_{2}-\chi_{2}\right) \cdots\left(\alpha_{r-1}-\chi_{r-1}\right) \quad(\bmod m) \\
& \equiv 1+\left(\alpha_{0}-1\right)+\left(\alpha_{0}-1\right) \sum_{r=2}^{j+1}\left(\alpha_{1}-\chi_{1}\right)\left(\alpha_{2}-\chi_{2}\right) \cdots\left(\alpha_{r-1}-\chi_{r-1}\right) \quad(\bmod m) \\
& \equiv \alpha_{0}+\left(\alpha_{0}-1\right) \sum_{i=1}^{j}\left(\alpha_{1}-\chi_{1}\right)\left(\alpha_{2}-\chi_{2}\right) \cdots\left(\alpha_{i}-\chi_{i}\right) \quad(\bmod m)
\end{aligned}
$$

As an example, let $m=5$ and $n=485=3 \cdot 5^{3}+4 \cdot 5^{2}+2 \cdot 5$. Then $j=3$ and the base 5 representation of 485 is $r_{5}(485)=\left(\alpha_{3}, \alpha_{2}, \alpha_{1}, \alpha_{0}\right)=(3,4,2,0)$. Therefore

$$
\chi_{3}=\chi_{2}=0, \chi_{1}=1
$$

and

$$
\begin{aligned}
c_{5}(5 \cdot 485) & \equiv-\left(\left(\alpha_{1}-1\right)+\left(\alpha_{1}-1\right) \alpha_{2}+\left(\alpha_{1}-1\right) \alpha_{2} \alpha_{3}\right) \quad(\bmod 5) \\
& =-(1+1 \cdot 4+1 \cdot 4 \cdot 3) \\
& =-17 \equiv 3 \quad(\bmod 5)
\end{aligned}
$$

In fact, we have $c_{5}(5 \cdot 485)=230358 \equiv 3(\bmod 5)$, which coincides with the above result.

To conclude this paper, we remark that the congruence (3.4) for $c_{m}(m n)$ is equivalent to Theorem 3.1 due to Andrews, Fraenkel and Sellers [4].

Proof of Theorem 3.1. Let $r_{m}(n)=\left(\alpha_{j}, \alpha_{j-1}, \ldots, \alpha_{1}, \alpha_{0}\right)$ be the base $m$ representation of $n$.

Following Lemma 2.9 of [4], we see that $c_{m}\left(m^{3} n\right) \equiv c_{m}(m n)(\bmod m)$ for all $n \geq 0$. Thereby to prove Theorem 3.1, it is sufficient to show the cases that $\ell=0$ and $\ell=1$, which correspond to $\alpha_{0}>0$ and $\alpha_{0}=0\left(\alpha_{1}>0\right)$, respectively.

If $\alpha_{0}>0$, then $\chi_{1}=0$. It leads to that

$$
c_{m}(m n) \equiv \alpha_{0}+\left(\alpha_{0}-1\right) \sum_{i=1}^{j} \alpha_{1}\left(\alpha_{2}-\chi_{2}\right) \cdots\left(\alpha_{i}-\chi_{i}\right) \quad(\bmod m) .
$$

We further consider the values of $\alpha_{1}, \alpha_{2}, \cdots, \alpha_{j}$. If $\alpha_{i}>0$ for $i \geq 1$, then $\chi_{i}=0$ for $2 \leq i \leq j$. Thus (3.5) turns to be

$$
c_{m}(m n) \equiv \alpha_{0}+\left(\alpha_{0}-1\right) \sum_{i=1}^{j} \alpha_{1} \cdots \alpha_{i} \equiv \alpha_{0}+\left(\alpha_{0}-1\right) \sum_{i=1}^{\infty} \alpha_{1} \cdots \alpha_{i} \quad(\bmod m)
$$


where $\alpha_{i}=0$ for $i>j$. Otherwise, suppose that $\alpha_{k}(1 \leq k \leq j)$ is the first zero in the sequence $\alpha_{1}, \alpha_{2}, \cdots$, then $\chi_{i}=0$ for $1 \leq i \leq k$. Noting that $\alpha_{k}=0$, we obtain

$$
\sum_{i=1}^{j} \alpha_{1}\left(\alpha_{2}-\chi_{2}\right) \cdots\left(\alpha_{i}-\chi_{i}\right)=\sum_{i=1}^{k-1} \alpha_{1} \alpha_{2} \cdots \alpha_{i}=\sum_{i=1}^{\infty} \alpha_{1} \alpha_{2} \cdots \alpha_{i}
$$

where $\alpha_{i}=0$ for $i>j$. Therefore, (3.5) leads to that

$$
c_{m}(m n) \equiv \alpha_{0}+\left(\alpha_{0}-1\right) \sum_{i=1}^{\infty} \alpha_{1} \cdots \alpha_{i} \quad(\bmod m)
$$

and both cases coincide with (3.1) with $\ell=0$.

If $\alpha_{0}=0$ and $\alpha_{1}>0$, following the same procedure, we obtain that

$$
\begin{aligned}
c_{m}(m n) & \equiv(-1) \sum_{i=1}^{j}\left(\alpha_{1}-1\right) \alpha_{2}\left(\alpha_{3}-\chi_{3}\right) \cdots\left(\alpha_{i}-\chi_{i}\right) \quad(\bmod m) \\
& \equiv 1-\alpha_{1}-\left(\alpha_{1}-1\right) \sum_{i=2}^{j} \alpha_{2}\left(\alpha_{3}-\chi_{3}\right) \cdots\left(\alpha_{i}-\chi_{i}\right) \quad(\bmod m) \\
& \equiv 1-\alpha_{1}-\left(\alpha_{1}-1\right) \sum_{i=2}^{\infty} \alpha_{2} \cdots \alpha_{i} \quad(\bmod m),
\end{aligned}
$$

which coincides with (3.2) with $\ell=1$. This completes the proof.

\section{Acknowledgments.}

This work was supported by the National Science Foundation of China, and the Natural Science Foundation of Tianjin, China.

\section{References}

[1] G.E. Andrews, Congruence properties of the $m$-ary partition function, J. Number Theory 3 (1971), 104-110.

[2] G.E. Andrews, E. Brietzke, Ø.J. Rødseth, and J.A. Sellers, Arithmetic properties of $m$-ary partitions without gaps, Ann. Combin. (2017), https://doi.org/10.1007/s00026-017-0369-6.

[3] G.E. Andrews, A.S. Fraenkel, and J.A. Sellers, Characterizing the number of $m$-ary partitions modulo $m$, Amer. Math. Monthly 122 (9) (2015), 880-885.

[4] G.E. Andrews, A.S. Fraenkel, and J.A. Sellers, $m$-ary partitions with no gaps: A characterization modulo $m$, Discrete Math. 339 (2016), 283-287. 
[5] G.E. Andrews and J.A. Sellers, On Sloane's generalization of non-squashing stacks of boxes, Discrete Math. 307 (2007), 1185-1190.

[6] R.F. Churchhouse, Congruence properties of the binary partition function, Math. Proc. Cambridge Philos. Soc. 66 (1969), 371-376.

[7] A. Folsom, Y. Homma, J.H. Ryu, and B. Tong, On a general class of non-squashing partitions, Discrete Math. 339 (2016), 1482-1506.

[8] H. Gupta, On m-ary partitions, Math. Proc. Cambridge Philos. Soc. 71 (1972), 343-345.

[9] M.D. Hirschhorn and J.H. Loxton, Congruence properties of the binary partition function, Math. Proc. Cambridge Philos. Soc. 78 (1975), 437-442.

[10] M.D. Hirschhorn and J.A. Sellers, A different view of $m$-ary partitions, Australas. J. Combin. 30 (2004), 193-196.

[11] Q.-H. Hou, H.-T. Jin, Y.-P. Mu, and L. Zhang, Congruences on the number of restricted $m$-ary partitions, J. Number Theory 169 (2016), 79-85.

[12] S. Ramanujan, Some properties of $p(n)$, the number of partitions of $n$, Math. Proc. Cambridge Philos. Soc. 19 (1919), 207-210.

[13] Ø.J. Rødseth, Some arithmetical properties of $m$-ary partitions, Math. Proc. Cambridge Philos. Soc. 68 (1970), 447-453.

[14] Ø.J. Rødseth and J.A. Sellers, On $m$-ary partition function congruences: A fresh look at a past problem, J. Number Theory 87 (2001), 270-281.

[15] N.J.A. Sloane and J.A. Sellers, On non-squashing partitions, Discrete Math. 294 (2005), 259-274. 